\title{
Efficacy of artemether-lumefantrine, the nationally-recommended artemisinin combination for the treatment of uncomplicated falciparum malaria, in southern Laos
}

Mayfong Mayxay ${ }^{1,2,3^{*}}$, Maniphone Khanthavong ${ }^{4}$, Odai Chanthongthip ${ }^{1}$, Mallika Imwong ${ }^{5}$, Tiengkham Pongvongsa ${ }^{6}$, Bouasy Hongvanthong ${ }^{4}$, Samalane Phompida ${ }^{4}$, Viengxay Vanisaveth ${ }^{4}$, Nicholas J White ${ }^{1,3,7}$ and Paul N Newton ${ }^{1,3}$

\begin{abstract}
Background: The Lao Government changed the national policy for uncomplicated Plasmodium falciparum malaria from chloroquine to artemether-lumefantrine (AL) in 2005. Since then, no information on AL efficacy has been reported. With evidence of resistance to artemisinin derivatives in adjacent Cambodia, there has been a concern as to AL efficacy. Monitoring of AL efficacy would help the Lao Government to make decisions on appropriate malaria treatment.
\end{abstract}

Methods: The efficacy of a three-day, twice daily oral artemether-lumefantrine for the treatment of uncomplicated falciparum malaria in Xepon District, Savannakhet Province, southern Laos was studied over 42 days follow-up. This was part of a trial of thiamin supplementation in falciparum malaria.

Results: Of 630 patients with P. falciparum enrolled in the trial of thiamin treatment, 549 (87\%, 357 children $\leq 15$ years and 192 adults) were included in this study. The per protocol 42-day cure rates were 97\% (524/541) [96\% (337/352) for children and 99\% (187/189) for adults, $p=0.042]$. By conventional intention-to-treat analysis, the 42-day cure rates adjusted for re-infection, were 97\% (532/549) [96\% (342/357) in children and 99\% (190/192) in adults, $p=0.042]$. The proportion of patients who remained parasitaemic at day 1 after treatment was significantly higher in children [33\% (116/356)] compared to adults [15\% (28/192)] $(p<0.001)$ and only one adult patient had detectable parasitaemia on day 2 . There were no serious adverse events. Potential side effects after treatment were reported more commonly in adults (32\%) compared to children $(15 \%)(p<0.001)$. Patients with recrudescent infections were significantly younger, had longer mean time to fever clearance, and had longer median time to parasite clearance compared to those who were cured.

Conclusions: The current nationally-recommended anti-malarial treatment (artemether-lumefantrine) remains highly efficacious for the treatment of uncomplicated falciparum malaria five years after introduction in Laos. Regular monitoring is required in case artemisinin-resistant $P$. falciparum parasites should appear.

Trial registration: ISRCTN85411059.

Keywords: Clinical trial, Plasmodium falciparum, Malaria, Artemisinin-based combination therapy (ACT) Artemether-lumefantrine, Coartem, Laos

\footnotetext{
* Correspondence: mayfong@tropmedres.ac

'Wellcome Trust-Mahosot Hospital-Oxford University Tropical Medicine

Research Collaboration, Mahosot Hospital, Vientiane, Lao PDR

${ }^{2}$ Faculty of Postgraduate Studies, University of Health Sciences, Vientiane

Lao PDR

Full list of author information is available at the end of the article
} 


\section{Background}

Although the incidence of Plasmodium falciparum malaria has declined recently in northern and central Lao PDR (Laos), malaria remains an important cause of morbidity and mortality in southern Laos, with an estimated incidence of 4.7 to $23.5 / 1,000$ population between 20062008 [1-3]. The change of the Lao Government national treatment policy for uncomplicated falciparum malaria from chloroquine to artemether-lumefantrine in 2005 is likely to have contributed substantially to the reduction in the incidence of malaria in northern and central Laos. This change was prompted by high levels of $P$. falciparum resistance to chloroquine (CQ) and sulphadoxine-pyrimethamine (SP) [4-8]. The use of artemisinin combination therapy (ACT), artesunate + mefloquine, artemether-lumefantrine (AL) and dihydroartemisininpiperaquine, has been shown to provide high cure rates (42-day failure rate of $\leq 6 \%$ ) and good tolerability in Laos [1,9-12].

However, artemisinin resistance has emerged in western Cambodia [13-15], raising serious concerns that resistance could spread the $\sim 400 \mathrm{~km}$ to southern Laos. In 2002-3, the 42-day cure rate following full treatment with AL was $97 \%$ in southern Laos despite a low-fat diet (which reduces lumefantrine absorption) and consequent low day 7 plasma lumefantrine concentrations [9]. AL has to be taken twice a day, and should be taken with fats [16-20] which may compromise adherence and efficacy respectively, so there is concern that AL efficacy could decline, particularly if protection from the coadministered artemether is compromised by emerging resistance.

Artemisinin resistance is manifest by a slowing in parasite clearance rates [15,21]. Reassuringly, of 861 patients with falciparum malaria recruited to three trials in southern Laos treated with ACT between 2002 and 2008 , only $2(0.23 \%)$ patients had parasite clearance times (PCT) longer than three days and $30(3.48 \%)$ had PCT longer than 2 days [22]. To determine whether there has been any recent change, the efficacy of the nationally recommended ACT (3-day oral artemetherlumefantrine) for the treatment of uncomplicated falciparum malaria was reported. This was part of a large trial of thiamin treatment in P. falciparum malaria in southern Laos, which will be reported separately.

\section{Methods}

\section{Study site, patients, clinical and laboratory procedures}

The study was conducted between June and November from 2008-2010 at Xepon (30 beds) Inter-District Hospital $\left(16.69^{\circ} \mathrm{N}, 106.20^{\circ} \mathrm{E}, 208\right.$ metres above sea level), Savannakhet Province, $\sim 665 \mathrm{~km}$ southeast of Vientiane, the capital of Laos. Xepon (88 villages, population 48,000 ) is inhabited predominantly by rice farmers of the Lao Theung ethnic groups. Malaria transmission is seasonal with a peak during the rainy months of July and August [22].

The clinical trial of thiamin supplementation in falciparum malaria conducted among patients with $P$. falciparum malaria receiving anti-malarial therapy according to national guidelines, was an exploratory, double-blind, parallel group, placebo-controlled, randomized (variable blocks), superiority trial to compare the frequency of clinical adverse effects and thiamin status, during 42 days follow up, of either daily oral thiamin supplementation ([oral thiamin (5 mg tablet) (Olan-Kemed Co.Ltd, Bangkok, Thailand)] $10 \mathrm{mg}$ immediately after anti-malarial drugs, followed by $10 \mathrm{mg}$ daily for 7 days followed by $5 \mathrm{mg}$ daily until day 42), or an identical placebo.

Patients were included in the trial of thiamin treatment provided that they or their guardians (in the case of children) gave fully informed written consent, were of any age, had microscopically confirmed $P$. falciparum infection or mixed Plasmodium species infections, with a history of fever, were willing and able to comply with the study protocol for the duration of the 42 days follow up, and had not taken a full course of any anti-malarial drugs in the previous three days. Patients were excluded if they had a history of hypersensitivity to thiamin or artemether-lumefantrine, presented with intercurrent non-malarial illness or any condition, which in the judgement of the investigator would place the subject at undue risk or interfere with the results of the study, and if they had clinically apparent suspected thiamin deficiency (beriberi) [23]. Patients included in the analysis for this paper were only those without danger signs or severe falciparum malaria [24], with $P$. falciparum mono- or mixed infections with other species, but an asexual $P$. falciparum parasite density of $<250,000 / \mu \mathrm{L}$, and were able to swallow artemether-lumefantrine at presentation.

Patients' admission clinical details including current and past medical history, pre-treatment, and findings from physical examination were recorded on the case record form. Venous blood samples were taken for parasite counts, haematocrit, blood glucose, and lactate, and three blood spots were collected on 3MM filter paper (Whatman, Maidstone, UK) and stored in a plastic bag with silica gel for later malaria genotype analysis [22,25]. For women of child bearing age a urinary pregnancy test was performed before anti-malarial treatment was prescribed.

If the study criteria were met, patients were admitted to the hospital and treated with the nationally recommended anti-malarial - artemether (20 mg)-lumefantrine (120 mg) (Co-artem ${ }^{\circledR}$, Novartis) provided by the Global Fund to fight AIDS, Tuberculosis and Malaria: one dose twice daily for three days. Dosing by body weight were 
one tablet if $<15 \mathrm{~kg}$, two tablets if $15-24 \mathrm{~kg}$, three tablets if $25-34 \mathrm{~kg}$, and four tablets if $\geq 35 \mathrm{~kg}$. All patients were asked to take fatty food with the antimalarial drugs and drug administration was directly observed by a study nurse. For children who could not swallow tablets, the appropriate drug dose was crushed and mixed with water and given in a syringe. If vomiting occurred within 30 minutes of anti-malarial administration, the full dose was repeated. Patient who vomited within 30 minutes to one hour, a half dose was readministered. Patients who vomited $>2$ times within one hour were given the rescue treatment (artesunate $2.4 \mathrm{mg} / \mathrm{kg}$ IV stat, followed by $2.4 \mathrm{mg} / \mathrm{kg}$ IV at 12 hours and 24 hours and then daily until able to take oral medication).

Vital signs (axillary temperature, pulse, respiratory rate, and blood pressure) were measured every six hours. Patients and parasite counts were reviewed daily until two consecutive negative blood smears, then weekly for 42 days from the start of treatment or at other times if they felt unwell. Patients were discharged only when their fever and parasitaemia had cleared (defined as axillary temperature $<37.5^{\circ} \mathrm{C}$ and $<1$ parasite per 500 white cells on thick film after 2 negative slides, respectively) and were asked to return to the hospital at days 7,14 , $21,28,35$ and 42 (or at any time the patients feel unwell) or were visited at home in case the patients did not return as planned.

At each weekly visit, the symptoms of the patients during the previous week were recorded. Finger-prick blood samples were taken for malaria blood smears and haematocrit and blood spots were collected onto filter paper strips from all patients with recurrent fever or malaria symptoms. PCR amplification was performed on paired samples for parasite genotyping to distinguish between reinfection and recrudescence using three parasite loci (MSP-1, MSP-2, and GLURP) [25]. The PCR tests were performed at the MORU malaria molecular laboratory, Bangkok, Thailand.

Patients with $P$. falciparum recurrence were retreated with artesunate $4 \mathrm{mg} / \mathrm{kg} /$ day in a single daily dose for 3 days (Day 0-2) (Guilin Pharmaceutical Co., Guilin, PRC) plus mefloquine $15 \mathrm{mg}$ base $/ \mathrm{kg}$ on day 1 and $10 \mathrm{mg}$ base/kg on day 2 in single daily dose (Lariam ${ }^{\mathrm{TM}}$, Roche Co, Switzerland). Those who had P. vivax appearance during follow up were treated with chloroquine (25 mg base $/ \mathrm{kg}$ ) for three days and further followed up. The incidence of any adverse event was recorded. Written informed consent was obtained from all participants.

Ethical clearance for the study was granted by the Lao National Ethics Committee for Health Research and the Oxford University Tropical Medicine Research Ethics Committee (OXTREC). The trial is registered with the ISRCTN via the University of Oxford as ISRCTN:
85411059. The trial was monitored by the Clinical Trials Support Group of Mahidol-Oxford Tropical Medicine Research Unit, Bangkok, Thailand.

\section{Outcome measures}

The primary objective of the thiamin treatment trial was to determine whether the frequency of adverse events, after anti-malarial therapy, were significantly lower in those who received thiamin supplementation in comparison to those who did not. Here the PCR-corrected adequate clinical and parasitological responses at day 42 [26], the PCT (the time in hours from the first treatment dose to the first of two consecutive thick films that were negative for asexual falciparum parasites after checking $\geq 500$ oil fields) and fever clearance times (FCT, time in hours from the start of treatment at which the tympanic temperature first dropped below $37.5^{\circ} \mathrm{C}$ and remained below $37.5^{\circ} \mathrm{C}$ for 48 hours), gametocytaemia, the frequency of adverse events, and changes in haematocrit following anti-malarial treatment are described.

\section{Statistical analysis}

Data were analysed using Stata v9 (StataCorp, College Station, TX, USA). Comparisons between two groups were made by the Mann-Whitney $U$, Student's $t$, chisquare, and Fisher's exact tests, as appropriate. Cure rates were calculated as the proportion of patients with PCR-confirmed recrudescence using intention-to-treat (ITT) and per-protocol (PP) populations and by KaplanMeier survival analysis. In the ITT population all losses to follow-up were treated as failures and in the PP population losses to follow-up were excluded from the analysis. Patients with new infections were regarded as cures in both analyses. Gametocyte carriage was summarized as person-gametocytes-week rates calculated as the total number of weeks with gametocytes divided by the total number of weeks of follow-up. To identify independent predictors of treatment response, a stepwise logistic regression was constructed with recrudescence as the dependent variable and sex, age, admission symptoms (headache, chill, myalgia, dizziness, vertigo, nausea, vomiting, abdominal pain, diarrhea, anorexia, cough, dyspnea, and weakness) and signs (drowsiness, splenomegaly, and hepatomegaly), gametocyte carriage at admission, admission temperature, baseline haematocrit, fever and parasite clearance times, past history of malaria, and $P$. vivax appearance during follow up included as covariates. Only factors that were significant at $\mathrm{p}<0.05$ were retained in the final model.

\section{Results}

Of the 630 patients enrolled, 549 (87\%; 276 randomized to thiamin and 273 to identical placebo) were included in the analysis for this paper. Patients were excluded 
$(\mathrm{N}=81)$ if they had either severe disease [24] $(\mathrm{n}=34)$ or parasitaemia $>250,000 / \mu \mathrm{L}(\mathrm{n}=27)$ or were not able to swallow tablets on admission $(\mathrm{n}=20) \quad$ (see above, Figure 1). As the median (range) PCT (days) and mean (95\%CI) FCT (hours) did not differ significantly between patients with and without thiamin supplementation [1 (1-3) vs 1 (1-3), $\mathrm{p}=0.87$; and $26.6(24.7-28.6)$ vs 26.4 (24.4-28.4), $\mathrm{p}=0.85$, respectively] all 549 patients were included in a single group. Admission and follow-up haematocrit and gametocytaemia also did not significantly differ between the two trial groups ( $\mathrm{p}>0.05)$.

Of the 549 patients (Figure 1), 538 (98\%) had P. falciparum mono-infection and 11 (2\%) had mixed infections (10 P. falciparum $+P$. vivax and $1 P$. falciparum $+P$. malariae), and 357 (65\%) were children aged $\leq 15$ years (Table 1). One child with persistent vomiting after taking AL was given parenteral artesunate and excluded from the study. Four patients (two children and two adults) were lost to follow up and three (two children and one adult) withdrew their consent for further follow up. Therefore, 352 (99\%) and 189 (98\%) children and adults, respectively, completed 42 days follow up (Figure 1).

\section{Demographic and clinical details}

All patients had a history of fever before admission and $402(73 \%)$ were febrile at the time of enrollment. The proportion of patients without documented fever on admission was similar between children and adults

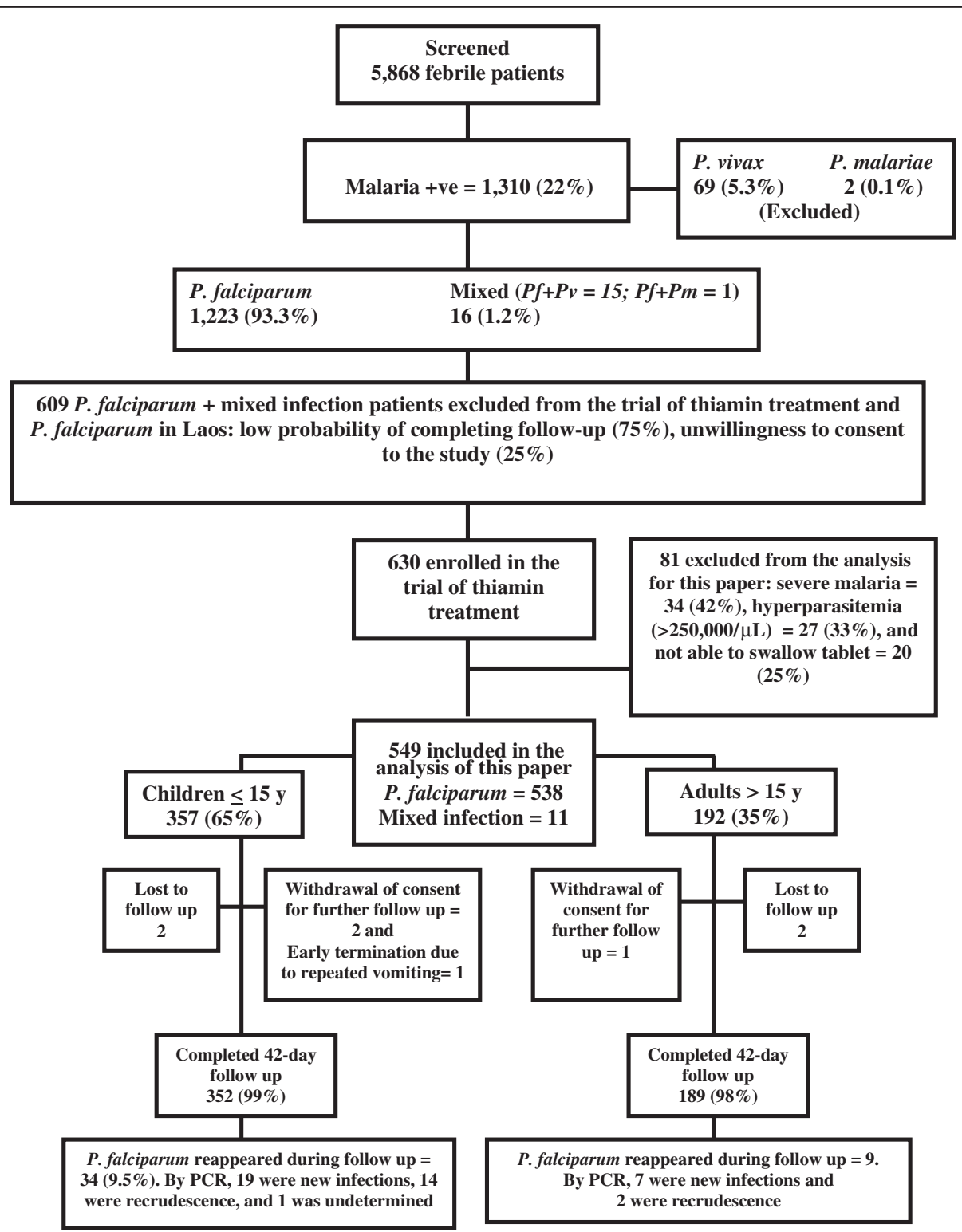

Figure 1 Patient flow diagram. 
Table 1 Admission demographic, clinical and laboratory details for patients included in the analysis of the efficacy of artemether-lumefantrine $\left(\right.$ Coartem $\left.^{\mathrm{TM}}\right)$ for the treatment of uncomplicated falciparum malaria in Laos

\begin{tabular}{|c|c|c|c|}
\hline \multirow[t]{2}{*}{ Variables } & \multicolumn{3}{|c|}{ Age groups } \\
\hline & All $(n=549)$ & Children $(\leq 15$ yrs $)(n=357)$ & Adults $(>15$ yrs $)(n=192)$ \\
\hline Sex, M, no (\%) & $276(50)$ & $174(49)$ & $102(53)$ \\
\hline Age, years, median (range) & $10(0.5-73)$ & $6(0.5-15)$ & $25(16-73)$ \\
\hline Body weight, kg & $29.5(28.0-30.9)$ & $18.8(17.8-19.8)$ & $49.3(48.2-50.4)$ \\
\hline Height, cm & $124.5(122.0-126.9)$ & $108.4(106.0-110.7)$ & $154.4(153.3-155.5)$ \\
\hline Previous malaria attack, no. (\%) of patients ${ }^{a}$ & $151(27.5)$ & $89(25)$ & $62(32)$ \\
\hline Axillary temperature, ${ }^{\circ} \mathrm{C}$ & $38.4(38.3-38.5)$ & $38.5(38.4-38.7)^{*}$ & $38.2(38.0-38.3)$ \\
\hline Patients without fever on admission, no. (\%) & $147(27)$ & $87(24)$ & $60(31)$ \\
\hline Systolic blood pressure, $\mathrm{mm} \mathrm{Hg}$ & $101.9(100.8-103.1)$ & $95.1(94.2-96.1)^{*}$ & $114.6(113.0-116.1)$ \\
\hline Diastolic blood pressure, $\mathrm{mm} \mathrm{Hg}$ & $69.7(68.8-70.6)$ & $64.6(63.7-65.5)^{*}$ & $79.3(78.2-80.4)$ \\
\hline Pulse, beats/min & $95.1(94.0-96.2)$ & $100.4(99.0-101.8)^{*}$ & $85.3(84.4-86.3)$ \\
\hline Respiratory rate/min & $27.5(27.0-28.1)$ & $29.7(29.0-30.3)^{*}$ & $23.5(22.9-24.0)$ \\
\hline Splenomegaly, no. (\%) of patients & $140 / 547(26)$ & $123 / 355(35)^{*}$ & 17/192 (9) \\
\hline Hepatomegaly, no (\%) of patients & $116 / 547(21)$ & $112 / 355(31.5)^{*}$ & $4 / 192(2)$ \\
\hline Lymphadenopathy, no (\%) of patients & $2 / 545(0.4 \%)$ & $2 / 356(0.6 \%)$ & 0 \\
\hline Parasitaemia, geometric mean parasites/ $\mu \mathrm{L}(95 \% \mathrm{Cl})$ & $19,775(17,244-22,678)$ & $27,241^{*}(23,281-31,875)$ & $10,902(8,567-13,872)$ \\
\hline Mixed malaria species infection, no.(\%) & $11(2)$ & $8(2)$ & $3(1.5)$ \\
\hline Gametocytaemia, no. (\%) of patients & $23(4)$ & $19(5)$ & $4(2)$ \\
\hline Gametocytaemia, geometric mean parasite/ $\mu \mathrm{L}(95 \% \mathrm{Cl})$ & $216(110-423)$ & $201(97-415)$ & $328(13-8,195)$ \\
\hline Haematocrit, \% & $35.8(35.2-36.3)$ & $33.7(33.1-34.4)^{*}$ & $39.5(38.7-40.4)$ \\
\hline Glucose, mmol/L & $6.09(5.97-6.20)$ & $6.09(5.93-6.24)$ & $6.08(5.90-6.27)$ \\
\hline Lactate, $\mathrm{mmol} / \mathrm{L}$ & $2.9(2.8-3.0)$ & $3.1(2.9-3.2)^{*}$ & $2.8(2.6-2.9)$ \\
\hline
\end{tabular}

NOTE. Data are presented as mean values $(95 \% \mathrm{Cl})$, unless otherwise indicated.

* Significantly different from the other group $(p<0.001)$.

${ }^{a}$ Defined as patient or patient's guardian reporting that the patient had had a febrile illness with a positive malaria slide.

(Tables 1 and 2). The admission mean temperature and geometric mean parasitaemia were significantly higher in children compared to adults, as were the proportions of patients with nausea, vomiting, irritability, or a palpable spleen or liver at presentation. The frequencies of headache, chill, myalgia, tinnitus, palpitations, and dyspnoea were significantly lower in children compared with adults (Table 2).

Fever and parasite clearance, and changes in haematocrit At presentation, $73 \%$ of all patients were febrile, (body temperature $\geq 37.5^{\circ} \mathrm{C}$ ), while at day $2,92 \%$ and $98 \%$ of children and adults were afebrile $(\mathrm{p}=0.004)$. The proportion of patients who remained parasitaemic at day 1 after treatment was significantly higher in children (33\%) compared to adults $(15 \%)(\mathrm{p}<0.001$, Table 3$)$ and only one adult patient had parasites detected at day 2. The mean FCT and median PCT were significantly longer in children than in adults (Table 3). The mean haematocrit at all time points (before and after treatment) was significantly lower in children compared to adults. Baseline (day 0) and day 28 mean haematocrits improved significantly after treatment for both children and adults (Paired $t$-test $\mathrm{p}<0.001$ for both groups, Table 3 ).

\section{Plasmodium falciparum gametocyte carriage}

Twenty-three (4\%) patients presented with patent gametocytaemia on admission, 19 (5\%) in children and 4 (2\%) in adults $(\mathrm{p}=0.07)$. The admission geometric mean gametocytaemia were similar for children and adults $(p=0.60)$. The time to clearance of gametocytes was longer in adults than in children but the difference was not statistically significant $(p=0.07)$. After treatment four children, but no adults, developed gametocytaemia (all at day 1) without gametocytaemia on admission. The median gametocyte-person-weeks was similar for children and adults (Table 3).

\section{Cure rates}

Of $43(7.8 \%)$ patients with subsequent $P$. falciparum reappearance (34 children, nine adults) PCR genotyping indicated that among these recurrent infections 16/43 (37\%) [14/34 (41\%) and 2/9 (22\%) children and adults, 
Table 2 Admission symptoms and signs for patients included in the analysis of the efficacy of artemether-lumefantrine $\left(\right.$ Coartem $\left.^{\mathrm{Tm}}\right)$ for the treatment of uncomplicated falciparum malaria in Laos

\begin{tabular}{|c|c|c|c|c|}
\hline \multirow[t]{2}{*}{ Symptoms and signs } & \multirow[t]{2}{*}{ Total $(n=549)$} & \multicolumn{3}{|c|}{ Age groups } \\
\hline & & Children $(\leq 15$ years $)(n=357)$ & Adults $(>15$ years) $(n=192)$ & $\mathrm{p}$-value \\
\hline Headache* & $372 / 404(92)$ & 189/212 (89) & 183/192 (95) & 0.02 \\
\hline Chill & 498/549 (91) & $316 / 357(88.5)$ & 182/192 (95) & 0.01 \\
\hline Myalgia* & 230/404 (57) & $75 / 212(35)$ & 155/192 (81) & $<0.001$ \\
\hline Weakness & $523 / 549$ (95) & 337/357 (94) & 186/192 (97) & 0.19 \\
\hline Dizziness* & 299/404 (74) & $150 / 212(71)$ & 149/192 (78) & 0.11 \\
\hline Vertigo* & $112 / 404(28)$ & $59 / 212(28)$ & $53 / 192(28)$ & 0.96 \\
\hline Tinnitus * & $83 / 404(20.5)$ & $24 / 212(11)$ & $59 / 192(31)$ & $<0.001$ \\
\hline Anorexia & $490 / 549(89)$ & $323 / 357(90)$ & 167/192 (87) & 0.20 \\
\hline Nausea* & $268 / 405(66)$ & $158 / 213(74)$ & $110 / 192(57)$ & $<0.001$ \\
\hline Vomiting & 296/549 (54) & 211/357 (59) & 85/192 (44) & 0.001 \\
\hline Abdominal pain* & $110 / 411(27)$ & $59 / 219(27)$ & $51 / 192(26.5)$ & 0.93 \\
\hline Diarrhoea & $111 / 548(20)$ & $72 / 356(20)$ & $39 / 192(20)$ & 0.98 \\
\hline Insomnia* & $313 / 549(57)$ & 203/357 (57) & $110 / 192(57)$ & 0.92 \\
\hline Nightmare* & $38 / 404(9)$ & 17/212 (8) & 21/192 (11) & 0.31 \\
\hline Palpitation* & 173/404 (43) & $62 / 212(29)$ & $111 / 192(58)$ & $<0.001$ \\
\hline Dyspnea* & $141 / 546(26)$ & $76 / 354(21)$ & 65/192 (34) & 0.002 \\
\hline Cough & $125 / 549(23)$ & $76 / 357(21)$ & 49/192 (25.5) & 0.25 \\
\hline Sore throat* & 49/410 (12) & 29/218 (13) & 20/192 (10) & 0.36 \\
\hline Irritable & 10/547 (2) & 10/355 (3) & 0 & 0.01 \\
\hline Rash & $1 / 549(0.2)$ & $1 / 357(0.3)$ & 0 & 1.00 \\
\hline Urticaria & $4 / 549(0.7)$ & 2/357 (0.6) & 2/192 (1) & 0.61 \\
\hline Itch & $4 / 549(0.7)$ & 2/357 (0.6) & 2/192 (1) & 0.61 \\
\hline Chest abnormality** & 2/549 (0.4) & $1 / 357(0.3)$ & $1 / 192(0.5)$ & 1.00 \\
\hline
\end{tabular}

NOTE. Data are shown as number (\%).

* Only children aged $\geq 4$ years were asked about these symptoms.

** Chest abnormality: crepitation, rhonchi.

respectively $(\mathrm{p}=0.44)$ ] had recrudescent infections. One child with $P$. falciparum reappearance had undetermined PCR result and, considering this child as having a recrudescent infection, the overall 42-day cure rates per protocol, excluding patients who were lost to follow up, withdrew consent, had persistent vomiting, or re-infection, were 97\% (524/541) [96\% (337/352) and 99\% (187/ 189) for children and adults, respectively, $\mathrm{p}=0.042]$. When the patients with repeated vomiting, who were lost to follow up or were withdrawn (censored at the time last seen), considering the child with undetermined PCR result as recrudescence, and regarding recrudescent infections as failures, the 42-day cure rates $(95 \% \mathrm{CI})$ by survival analysis (Kaplan-Meier) were 99.0\% (98.3 99.4\%) for children and $99.8 \%(99.0$ - 99.9\%) for adults. For the PCR adjusted survival analysis, the cure rates $(95 \% \mathrm{CI})$ were $99.0 \%(98.3$ - 99.4\%) and 99.8\% (99.0 99.9\%) for children and adults, respectively. By conventional ITT analysis, the overall 42-day cure rates, adjusted for re-infection, were 97\% (532/549) [96\% (342/ $357)$ in children and 99\% (190/192) in adults, $\mathrm{p}=0.042]$. Of 17 patients considered as having recrudescent infection (15 children and two adults), 15 (13 children and two adults) had parasite recurrence at or before day 28 . Similarly, the 28-day cure rates by ITT analysis were 344/357 (96\%) for children and 190/192 (99\%) for adults. The median (range) interval to recrudescent falciparum infections was 21 (14-42) days. By ITT and per protocol analyses, the 42-day PCR uncorrected cure rates for children and adults were 323/357 (90\%) vs 183/192 (95\%); $(\mathrm{p}=0.04)$, and $323 / 352 \quad(92 \%)$ vs $183 / 189 \quad(97 \%)$ $(\mathrm{p}=0.02)$, respectively. Of 129 children aged $<5$ years, six had recrudescent infection and one had undetermined PCR result. If the patient with undetermined PCR result was considered a recrudescent infection, the 42day PCR corrected cure rates among children $<5$ years 
Table 3 Outcome measures for the treatment of patients included in the analysis of the efficacy of artemetherlumefantrine $\left(\right.$ Coartem $\left.^{\mathrm{TM}}\right)$ in the treatment of uncomplicated falciparum malaria in Laos

\begin{tabular}{|c|c|c|c|}
\hline \multirow[t]{2}{*}{ Variables } & \multicolumn{3}{|c|}{ Age groups } \\
\hline & All $(n=549)$ & Children (age $\leq 15$ yrs) $(n=357)$ & Adults (age $>15$ yrs) $(n=192)$ \\
\hline 42-day cure rate, no. (\%) of patients ${ }^{a}$ & $532 / 549(97)$ & $342 / 357(96)^{*}$ & 190/192 (99) \\
\hline 42-day cure rate per protocol, no. (\%) of patients $\psi$ & $524 / 541(97)$ & $337 / 352(96)^{*}$ & 187/189 (99) \\
\hline Fever clearance time, mean hours $(95 \% \mathrm{Cl})^{\mathrm{a}, \mathrm{b}}$ & $25.3(23.9-26.8)$ & $27.6(25.7-29.5)^{*}$ & $21.2(19.2-23.2)$ \\
\hline Patients remained febrile at day 1 , no. (\%) & $241(44)$ & $169(47)^{*}$ & $72(37.5)$ \\
\hline Patients remained febrile at day 2, no. (\%) & $33(6)$ & $29(8)^{*}$ & $4(2)$ \\
\hline Parasite clearance time, median days (range) $)^{a, c}$ & $1(1-3)$ & $1(1-2)^{*}$ & $1(1-3)$ \\
\hline Positive parasitaemia at day 1 , no. (\%) of patients & $144 / 548(26)$ & $116 / 356(33)^{*}$ & 28/192 (15) \\
\hline Positive parasitaemia at day 2 , no. (\%) of patients & 1 & 0 & 1 \\
\hline Gametocytaemia detected at anytime, no.(\%) of patients & $27(5)$ & $23(6)$ & $4(2)$ \\
\hline Gametocytaemia after treatment, no. (\%) of patients & $4(0.73)$ & $4(1.12)$ & 0 \\
\hline Gametocyte clearance time, median days (range) $^{d}$ & $7(1-21)$ & $3(1-21)$ & $7(7-14)$ \\
\hline Gametocyte cleared by day 7, no. (\%) ${ }^{d}$ & $24(89)$ & $21(91)$ & $3(75)$ \\
\hline Median (range) gametocyte-person-weeks ${ }^{d}$ & $0.42(0.14-1.0)$ & $0.42(0.14-1.0)$ & $0.42(0.42-0.42)$ \\
\hline $\begin{array}{l}\text { P. vivax appearance after treatment for } \\
\text { P. falciparum, no. (\%) of patients }\end{array}$ & 18/594 (3) & $17 / 357(5)^{*}$ & $1 / 192(0.5)$ \\
\hline Day 0 haematocrit, mean \% (95\%Cl) & $35.8(35.2-36.3)$ & $33.7(33.1-34.4)^{*}$ & $39.5(38.7-40.4)$ \\
\hline Day 1 haematocrit, mean \% (95\%Cl) & $32.4(31.9-32.9)$ & $30.4(29.8-30.9)^{*}$ & $36.2(35.4-36.9)$ \\
\hline Day 2 haematocrit, mean \% (95\%Cl) & $31.1(30.6-31.6)$ & $29.1(28.5-29.6)^{*}$ & $34.9(34.1-35.7)$ \\
\hline Day 3 haematocrit, mean \% (95\%Cl) & $31.1(30.6-31.6)$ & $29.0(28.5-29.6)^{*}$ & $34.9(34.1-35.6)$ \\
\hline Day 7 haematocrit, mean \% (95\%Cl) & $33.3(32.9-33.7)$ & $31.7(31.2-32.1)^{*}$ & $36.3(35.6-37.0)$ \\
\hline Day 14 haematocrit, mean \% (95\%Cl) & $34.4(34.0-34.8)$ & $33.3(32.9-33.7)^{*}$ & $36.5(35.8-37.2)$ \\
\hline Day 21 haematocrit, mean \% (95\%Cl) & $35.6(35.2-35.9)$ & $34.6(34.2-35.0)^{*}$ & $37.5(36.8-38.2)$ \\
\hline Day 28 haematocrit, mean \% $(95 \% \mathrm{Cl})$ & $36.2(35.8-36.5)$ & $35.3(34.9-35.7)^{*}$ & $37.8(37.0-38.5)$ \\
\hline Day 35 haematocrit, mean \% (95\%Cl) & $36.5(36.1-36.8)$ & $35.5(35.2-35.9)^{*}$ & $38.2(37.5-38.9)$ \\
\hline Day 42 haematocrit, mean \% $(95 \% \mathrm{Cl})$ & $37.6(37.2-37.9)$ & $36.7(36.3-37.0)^{*}$ & $39.4(38.7-40.1)$ \\
\hline
\end{tabular}

NOTE. $\Psi$ The patient with undetermined PCR result was classified as recrudescent infection.

${ }^{a}$ Intention-to-treat analysis.

b Data were available from 340 and 184 patients in children and adults groups, respectively.

' Data were available from 356 and 192 patients in children and adults groups, respectively.

d Data were available from 23 and 4 patients in children and adults groups, respectively.

* Significant difference from the other group $(p<0.05)$.

were $95 \%$. There were 18 episodes of vivax malaria during the follow-up period, of which 17 were in children (p $<0.05$ ). The median (range) interval to $P$. vivax appearance was 28 (21-42) days (Table 3$)$.

\section{Factors affecting treatment response}

Patients with recrudescent infections were significantly younger [median (range) age $5(2-25)$ years], than those whose infections were treated successfully [median (range) age $10(0.5-73)$ years, $\mathrm{p}=0.006]$. Considering the child with undetermined PCR result as recrudescent infection, the proportion of treatment failures adjusted for re-infection by PCR among children was 4.2\% (15/357 patients) compared with $1.0 \%$ (2/192 patients) among adults $(\mathrm{p}=0.042)$. The admission mean $(95 \% \mathrm{CI})$ body temperature was significantly higher in patients with recrudescent infections $\left[39.1(38.4-39.8)^{\circ} \mathrm{C}\right]$ than those who were cured [38.4 $\left.(38.3-38.5){ }^{0} \mathrm{C}, \mathrm{p}=0.01\right]$. Patients with recrudescent infections had a significantly longer mean FCT $(45.0 \mathrm{~h} ; 95 \% \mathrm{CI}, 32.3-57.8 \mathrm{~h})$ than those patients without $(24.8 \mathrm{~h} ; 95 \% \mathrm{CI}, 23.4-26.2 \mathrm{~h} ; \mathrm{p}<0.001)$. Patients with recrudescent infections also had a significantly lower haematocrit at days $3,14,21,28$, and 35 after starting therapy than those without recrudescence detected $(\mathrm{p}=0.03, \mathrm{p}=0.01, \mathrm{p}<0.001, \mathrm{p}<0.001$, and $\mathrm{p}<0.001$, respectively). The proportion of the patients reporting a past history of malaria was significantly lower in the patients with recrudescent infection (6\%) compared to those without $(28 \%)(\mathrm{p}=0.043)$. In a multiple logistic regression analysis, admission high 
temperature $[\mathrm{OR}=2.2, \quad 95 \% \mathrm{CI}=1.2-4.2), \mathrm{p}=0.01]$, longer fever clearance times $[\mathrm{OR}=1.0,95 \% \mathrm{CI}=1.0-1.1$, $\mathrm{p}=0.01]$, and lower haematocrit at day $28[\mathrm{OR}=0.7,95 \%$ $\mathrm{CI}=0.6-0.9, \mathrm{p}=0.005]$ were significantly associated with treatment failure.

\section{Adverse events}

The possible adverse events following treatment with AL are shown in Table 4. Adverse event symptoms were recorded for only those 467 (85\%) patients aged $\geq 4$ years old and able to answer questions. No serious adverse events including deaths were found during follow up. The proportion of patients with at least one recorded potential side effect after treatment was significantly higher in adults (32\%) compared to children (15\%) ( $p<0.001)$, but this could be due to difficulties of obtaining histories from children. The incidence of posttreatment insomnia, weakness, anorexia, diarrhoea, nightmares and palpitation were all significantly higher in adults compared to children $(\mathrm{p}<0.05)$ (Table 4$)$.

\section{Discussion}

This large clinical trial shows that artemetherlumefantrine retains excellent efficacy in the treatment of uncomplicated falciparum malaria at one site in southern Laos in both children and adults with 42-days PCR-corrected cure rates of $96 \%$ and $99 \%$, respectively. These results are similar to those described in a comparable trial conducted in 2002-3 in nearby Phalanxay District ( $60 \mathrm{Km}$ west of Xepon), in which there was a 42-days cure rate of $97 \%$ in children and adults [9]. A similar trial conducted in the northern Luang Namtha Province of Laos in 2003 also showed very good efficacy and tolerability of AL with a 42-days cure rate of 94\% [11]. As previously, AL was very well tolerated, proved safe and no serious adverse events were found during follow up.

Patients' background immunity contributes substantially to the treatment response of uncomplicated malaria in Laos $[6,27]$. In this study, children had significantly higher recrudescence rates and $P$. vivax appearance rates than adults. A study comparing oral chloroquine and sulphadoxinepyrimethamine for the treatment of uncomplicated falciparum malaria, before the introduction of AL, found treatment failure rates among Lao children to be 4.9 times higher than those among adults [6]. The contribution of immunity to the treatment response is also suggested by slower clearance of fever and parasitaemia among children

Table 4 Possible adverse events (AE) found in patients included in the analysis of the efficacy of artemetherlumefantrine $\left(\right.$ Coartem $\left.^{\mathrm{TM}}\right)$ in the treatment of uncomplicated falciparum malaria in Laos*

\begin{tabular}{|c|c|c|c|c|}
\hline \multirow[t]{2}{*}{ Variables } & \multicolumn{4}{|c|}{ Age groups } \\
\hline & All $(N=549)$ & Children (age $\leq 15$ years) $(n=357)$ & Adults (age $>15$ years) $(n=192)$ & p-value \\
\hline At least one adverse event & $114 / 549(21 \%)$ & $52 / 357(15 \%)$ & $62 / 192(32 \%)$ & $<0.001$ \\
\hline Headache & $32 / 403(8 \%)$ & $13 / 211(6 \%)$ & 19/192 (10\%) & 0.16 \\
\hline Insomnia & $31 / 549(6 \%)$ & $14 / 357(4 \%)$ & $17 / 192(9 \%)$ & 0.01 \\
\hline Weakness & $29 / 549(5 \%)$ & $12 / 357(3 \%)$ & $17 / 192(9 \%)$ & 0.006 \\
\hline Anorexia & $30 / 549(5 \%)$ & $14 / 357(4 \%)$ & 16/192 (8\%) & 0.03 \\
\hline Diarrhoea & $25 / 549(5 \%)$ & $11 / 357(3 \%)$ & $14 / 192(7 \%)$ & 0.02 \\
\hline Nightmare & $17 / 403(4 \%)$ & $4 / 211(2 \%)$ & $13 / 192(7 \%)$ & 0.02 \\
\hline Abdominal pain & $12 / 410(3 \%)$ & $5 / 218(3 \%)$ & $7 / 192(4 \%)$ & 0.42 \\
\hline Dizziness & $11 / 403(3 \%)$ & $4 / 211(2 \%)$ & 7/192 (4\%) & 0.36 \\
\hline Vomiting & $10 / 549(2 \%)$ & $8 / 357(2 \%)$ & 2/192 (1\%) & 0.32 \\
\hline Nausea & $5 / 403(1 \%)$ & $2 / 211(1 \%)$ & $3 / 192(2 \%)$ & 0.67 \\
\hline Palpitation & $5 / 404(1 \%)$ & 0 & $5 / 192(3 \%)$ & 0.02 \\
\hline Itch & $5 / 549(1 \%)$ & 3/357 (1\%) & 2/192 (1\%) & 1.00 \\
\hline Vertigo & $1 / 403(0.25 \%)$ & 0 & $1 / 192$ (0.5\%) & 0.47 \\
\hline Tinnitus & $1 / 404(0.25 \%)$ & 0 & $1 / 192(0.5 \%)$ & 0.47 \\
\hline Rash & $1 / 549(0.2 \%)$ & $1 / 357(0.3 \%)$ & 0 & 1.00 \\
\hline Urticaria & $1 / 549(0.2 \%)$ & 0 & $1 / 192(0.5 \%)$ & 0.35 \\
\hline Irritability & 0 & 0 & 0 & - \\
\hline Dyspnoea & 0 & 0 & 0 & - \\
\hline Hearing loss & 0 & 0 & 0 & - \\
\hline
\end{tabular}

* Symptoms are given for only those 467 patients aged $\geq 4$ years old and able to answer questions about these symptoms, except that parents/guardians were asked about their charges insomnia, weakness, anorexia and itch. 
compared to adults [1,9]. Anti-malarial drug assessments conducted only in adults, may overestimate anti-malarial efficacy risking continuation of ineffective drug regimens in national policy [28]. Anti-malarial pharmacokinetics may differ in children and but for artemether-lumefantrine these differences are small [29].

The evidence, both from a trial of artesunate monotherapy with a mean (95\%CI) PCT (hours) of 23.2 (21.2-25.3) and 42-day PCR-corrected cure rate of $100 \%$ [22], and from the high efficacy of AL described here, at the same site, suggests that artemisinin resistant $P$. falciparum has not spread to this area, of southern Laos. However, regular monitoring of the clinical efficacy of artemisinin-derivatives in southern Laos, especially closer to the Cambodian border, is required.

\section{Conclusion}

The Lao nationally recommended anti-malarial drug (artemether-lumefantrine) remains highly efficacious for the treatment of uncomplicated falciparum malaria five years after its introduction. However, regular monitoring of the efficacy of artemisinin derivatives is essential as early warning of the potential spread of artemisininresistant $P$. falciparum parasites.

\section{Competing interests}

The authors declare that they have no competing interests.

\section{Authors' contributions}

MM designed the study, recruited and followed up the patients, analysed data and drafted paper. MK and OC designed the study, recruited and followed up the patients, and revised the paper. MI designed the study, performed the molecular genetic studies, and revised the paper. TP, BH, W, SP and NJW designed the study and revised the paper. PNN designed the study, analysed data, drafted and revised the paper. All authors read and approved the final manuscript.

\section{Acknowledgements}

We are very grateful for the involvement of all patients in this study and for the technical help from Mrs. Manisack Phommasansack, Mr. Bounpone Phimphalat, Mrs. Phinnakone Lasachack, Mrs. Phanmala Baysy, Mrs. Ammala Phomsimone, Mrs. Bounmy Syphachanh, Mrs. Vilayphone, Mr. Chanthala Vilayhong, Mr. Phonepasit Panyanouvong, Mr. Sengchanh Yeuchaixiong, and all doctors, medical assistants and nurses in Xepon District Hospitals. We gratefully thank Sue Lee, Brian Angus, Francois Nosten, and Nick Anstey, for participating in the Data and Safety Monitoring Committee of this study and Liz Ashley and Francois Nosten for helpful comments on the ms. Our grateful thanks go to Phaik Yeong Cheah, Prayoon Yuentrakul, and Viriya Hantrakun for acting as external monitors and to Drs. Pranom Phongmany and Sengchanh Koumphiengmaniseng for their valuable advice. We are very grateful for the support of the Minister of Health, His Excellency Dr. Ponmek Dalaloy, the Directors of Hygiene and Preventive Medicine, Drs.

Douangchanh Keo-Asa and Bounlay Phommasack, the Director of Mahosot Hospital, Professor Chanpheng Thammavong. This study was supported by the Wellcome Trust of Great Britain.

\footnotetext{
Author details

${ }^{1}$ Wellcome Trust-Mahosot Hospital-Oxford University Tropical Medicine Research Collaboration, Mahosot Hospital, Vientiane, Lao PDR. ${ }^{2}$ Faculty of Postgraduate Studies, University of Health Sciences, Vientiane Lao PDR. ${ }^{3}$ Centre for Clinical Vaccinology and Tropical Medicine, Churchill Hospital, University of Oxford, Oxford, UK. ${ }^{4}$ Centre of Malariology,
}

Parasitology and Entomology, Vientiane, Lao PDR. ${ }^{5}$ Department of Molecular Tropical Medicine and Genetics, Faculty of Tropical Medicine, Mahidol University, Bangkok, Thailand. ${ }^{6}$ Savannakhet Provincial Malaria Station, Savannakhet Province, Lao PDR. 'Faculty of Tropical Medicine, Mahidol University, Bangkok, Thailand.

Received: 6 April 2012 Accepted: 24 May 2012

Published: 8 June 2012

\section{References}

1. Mayxay M, Thongpraseuth V, Khanthavong M, Lindegårdh N, Barends M, Keola S, Pongvongsa T, Phompida S, Phetsouvanh R, Stepniewska K, White NJ, Newton PN: An open, randomized comparision of artesunate plus mefloquine vs. dihydroartemisinin-piperaquine for the treatment of uncomplicated Plasmodium falciparum malaria in the Lao People's Democratic Republic (Laos). Trop Med Int Health 2006, 11:1157-1165.

2. Shirayama Y, Phompida S, Kuroiwa C: Monitoring malaria control in Khammouane Province, Laos: an active case detection survey of Plasmodium falciparum malaria using the Paracheck rapid diagnostic test. Trans R Soc Trop Med Hyg 2008, 102:743-750.

3. Jorgensen P, Nambanya S, Gopinath D, Hongvanthong B, Luangphengsouk K Bell D, Phompida S, Phetsouvanh R: High heterogeneity in Plasmodium falciparum risk illustrates the need for detailed mapping to guide resource allocation: a new malaria risk map of the Lao People's Democratic Republic. Malar J 2010, 9:59.

4. Pillai DR, Labbe AC, Vanisaveth V, Hongvanthong B, Phompida S, Inthakone S, Zhong K, Kain KC: Plasmodium falciparum malaria in Laos: chloroquine treatment outcome and predictive value of molecular markers. $J$ Infect Dis 2001, 183:789-795.

5. Guthmann JP, Kasparian S, Phetsouvanh R, Nathan N, Garcia M, Phompida S, Brockman A, Gastellu M, Legros D: The efficacy of chloroquine for the treatment of acute uncomplicated Plasmodium falciparum malaria in the Lao People's Democratic Republic. Ann Trop Med Parasitol 2002, 96:553-557.

6. Mayxay M, Newton PN, Khanthavong M, Tiengkham P, Phetsouvanh R, Phompida S, Brockman A, White NJ: Chloroquine versus sulphadoxinepyrimethamine for treatment of Plasmodium falciparum malaria in Savannakhet province, Lao People's Democratic Republic: an assessment of national antimalarial drug recommendations. Clin Infect Dis 2003, 37:1021-1028.

7. Mayxay M, Phetsouvanh R, Phompida S, Newton PN, Khanthavong M, Vannachone B, Brockmans A, White NJ: A randomized comparison of oral chloroquine and sulphadoxine-pyrimethamine for the treatment of uncomplicated falciparum malaria in Laos. Trans R Soc Trop Med Hyg 2003, 97:343-344.

8. Schwobel B, Jordan S, Vanisaveth V, Phetsouvanh R, Christophel EM, Phompida S, von Sonnenburg F, Jelinek T: Therapeutic efficacy of chloroquine plus sulphadoxine/pyrimethamine compared with monotherapy with either chloroquine or pyrimethamine/sulphadoxine in uncomplicated Plasmodium falciparum malaria in Laos. Trop Med Int Health 2003, 8:19-24.

9. Mayxay M, Khanthavong M, Lindegårdh N, Keola S, Barends M, Pongvongsa T, Yapom R, Annerberg A, Phompida S, Phetsouvanh R, White NJ, Newton PN: Randomized comparison of chloroquine plus sulphadoxine-pyrimethamine versus artesunate plus mefloquine versus artemether-lumefantrine in the treatment of uncomplicated falciparum malaria in the Lao PDR (Laos). Clin Infect Dis 2004, 39:1139-1147.

10. Mayxay M, Keomany S, Khanthavong M, Souvannasing P, Stepniewska K, Khomthilath T, Keola S, Pongvongsa T, Phompida S, Ubben D, Valecha N White NJ, Newton PN: A phase III, randomized, non-inferiority trial to assess the efficacy and safety of dihydroartemisinin-piperaquine in comparison with artesunate-mefloquine in patients with uncomplicated Plasmodium falciparum malaria in Southern Laos. Am J Trop Med Hyg 2010, 83:1221-1229.

11. Stohrer JM, Dittrich $\mathrm{S}$, Thongpaseuth V, Vanisaveth V, Phetsouvanh $\mathrm{B}$, Phompida S, Monti F, Christophel EM, Lindegardh N, Annerberg A, Jelinek T: Therapeutic efficacy of artemether-lumefantrine and artesunatemefloquine for treatment of uncomplicated Plasmodium falciparum malaria in LuangNamtha Province, Lao People's Democratic Republic. Trop Med Int Health 2004, 9:1175-1183. 
12. Valecha N, Phyo AP, Mayxay M, Newton PN, Krudsood S, Keomany S, Khanthavong M, Pongvongsa T, Ruangveerayuth R, Uthaisil C, Ubben D, Duparc S, Bacchieri A, Corsi M, Rao BHK, Bhattacharya PC, Dubhashi N, Ghosh SK, Vas Dev, Kumar A, Pukittayakamee S: An open-label, randomised study of dihydroartemisinin-piperaquine versus artesunate-mefloquine for falciparum malaria in Asia. PLoS One 2010, 5:e11880.

13. Noedl H, Se Y, Schaecher K, Smith BL, Socheat D, Fukuda MM: Artemisinin Resistance in Cambodia 1 (ARC1) Study Consortium. Evidence of artemisinin-resistant malaria in western Cambodia. N Engl J Med 2008, 359:2619-2620

14. Rogers WO, Sem R, Tero T, Chim P, Lim P, Muth S, Socheat D, Ariey F, Wongsrichanalai $C$ : Failure of artesunate-mefloquine combination therapy for uncomplicated Plasmodium falciparum malaria in southern Cambodia. Malar J 2009, 12:10

15. Dondorp AM, Nosten F, Yi P, Das D, Phyo AP, Tarning J, Lwin KM, Ariey F, Hanpithakpong W, Lee SJ, Ringwald P, Silamut K, Imwong M, Chotivanich K, Lim P, Herdman T, An SS, Yeung S, Singhasivanon P, Day NP, Lindegardh N, Socheat D, White NJ: Artemisinin resistance in Plasmodium falciparum malaria. N Eng J Med 2009, 361:455-467.

16. van Vugt M, Brockman A, Gemperli B, Luxemburger C, Gathmann I, Royce C, Slight T, Looareesuwan S, White NJ, Nosten F: Randomized comparison of artemether-benflumetol and artesunate-mefloquine in treatment of multidrug-resistant falciparum malaria. Antimicrob Agents Chemother 1998, 42:135-139.

17. van Vugt M, Wilairatana P, Gemperli B, Gathmann I, Phaipun L, Brockman A, Luxemburger C, White NJ, Nosten F, Looareesuwan S: Efficacy of six doses of artemether-lumefantrine (benflumetol) in multidrug-resistant Plasmodium falciparum malaria. Am J Trop Med Hyg 1999, 60:936-942

18. Lefevre G, Looareesuwan S, Treeprasertsuk S, Krudsood S, Silachamroon U, Gathmann I, Mull R, Bakshi R: A clinical and pharmacokinetic trial of six doses of artemether-lumefantrine for multidrug-resistant Plasmodium falciparum malaria in Thailand. Am J Trop Med Hyg 2001, 64:247-256

19. Poravuth $Y$, Socheat D, Fandeur T, Tsuyuoka R, Hoyer S: Efficacy and safety of six dose regimen of Coartem ${ }^{\circledR}$ in the treatment of uncomplicated falciparum malaria in Cambodia. Mekong Malaria Forum 2002, 10:54-55.

20. Krudsood S, Chalermrut K, Pengruksa C, Srivilairit S, Silachamroon U, Treeprasertsuk S, Kano S, Brittenham GM, Looareesuwan S: Comparative clinical trial of two-fixed combinations dihydroartemisin-napthoquinetrimethoprim (DNP) and artemether-lumefantrine (Coartem/Riamet) in the treatment of acute uncomplicated falciparum malaria in Thailand. Southeast Asian J Trop Med Public Health 2003, 34:316-321.

21. Flegg JA, Guerin PJ, White NJ, Stepniewska K: Standardizing the measurement of parasite clearance in falciparum malaria: the parasite clearance estimator. Malar J 2011, 10:339. http://www.malariajournal.com/ content/10/1/339.

22. Mayxay M, Khanthavong M, Chanthongthip O, Imwong M, Lee SJ, Stepniewska K, Soonthornsata B, Pongvongsa T, Phompida S, Hongvanthong B, Ringwald P, White NJ, Newton PN: No evidence for spread of Plasmodium falciparum artemisinin resistance to Savannakhet Province, Southern Laos. Am J Trop Med Hyg 2012, 86:403-408.

23. World Health Organization: Thiamine deficiency and its prevention and control in major emergencies. Geneva; 1999. WHO/NHD/99.13.

24. World Health Organization: Severe falciparum malaria. Trans $R$ Soc Trop Med Hyg 2000, 94(Supplement 1):1-90.

25. Brockman A, Paul REL, Anderson TJC, Hackford I, Phaiphun L, Looareesuwan S, Nosten F, Day KP: Application of genetic markers to the identification of recrudescent Plasmodium falciparum infections on the northwestern border of Thailand. Am J Trop Med Hyg 1999, 60:14-21.

26. World Health Organization: Method for surveillance of antimalarial drug efficacy. Geneva: World Health Organization; 2009. http://www.who.int/ malaria/resistance.

27. Mayxay M, Chotivanich K, Pukrittayakamee S, Newton P, Looareesuwan S, White NJ: The contribution of humoral immunity to the therapeutic response in falciparum malaria. Am J Trop Med Hyg 2001, 65:918-923.
28. White NJ: The assessment of antimalarial drug efficacy. Trends Parasitol 2002, 18:458-464

29. Tarning J, Zongo I, Somé FA, Rouamb N, Parikh S, Rosenthal PJ, Hanpithakpong W, Jongrak N, Day NP, White NJ, Nosten F, Ouedraogo JB, Lindegardh N: Population pharmacokinetics and pharmacodynamics of piperaquine in children with uncomplicated falciparum malaria. Clin Pharmacol Ther 2012, 91:497-505.

doi:10.1186/1475-2875-11-184

Cite this article as: Mayxay et al:: Efficacy of artemether-lumefantrine, the nationally-recommended artemisinin combination for the treatment of uncomplicated falciparum malaria, in southern Laos. Malaria Journal 2012 11:184.

\section{Submit your next manuscript to BioMed Central and take full advantage of:}

- Convenient online submission

- Thorough peer review

- No space constraints or color figure charges

- Immediate publication on acceptance

- Inclusion in PubMed, CAS, Scopus and Google Scholar

- Research which is freely available for redistribution 